SECTION 2. Applied mathematics. Mathematical modeling.

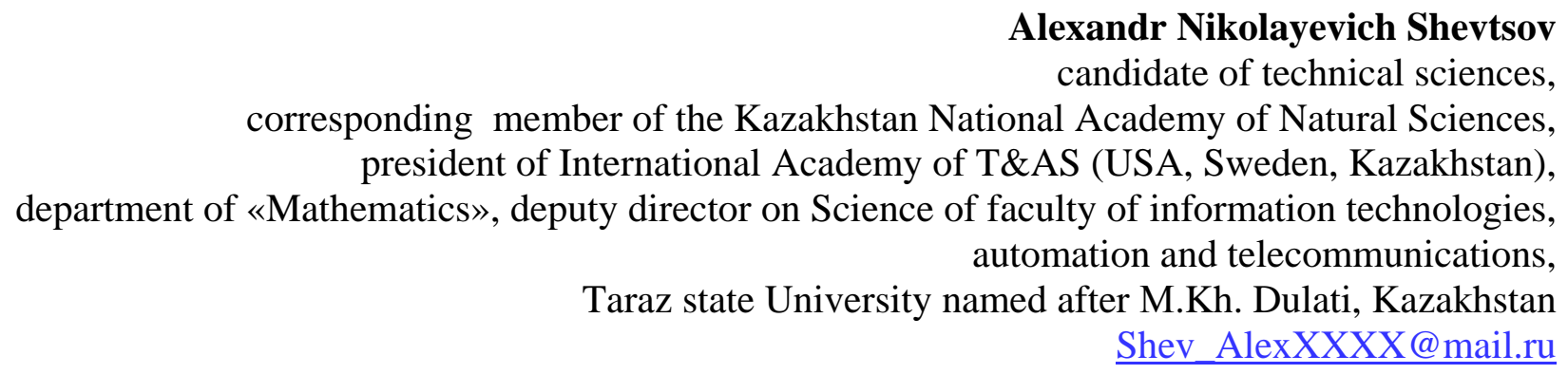

Nazgul Zhangaliyevna Yeskali

student, Department of Mathematics

Taraz state University named after M.Kh. Dulati, Kazakhstan

Aida Askarovna Sabdenbek

student, Department of Mathematics

Taraz state University named after M.Kh. Dulati, Kazakhstan

Meirkhat Maratovich Uakbayev

student, Department of Mathematics

Taraz state University named after M.Kh. Dulati, Kazakhstan

\title{
ABOUT THE PROBLEM OF ARBITRARY ALTERNATING PLUS AND MINUS SIGNS IN ARITHMETIC SERIES
}

\begin{abstract}
When finding the sum of a number and the $n$-th member of alternating series, there are several problems when the number is not snakecharmers. In the article are a few formulas to solve this problem, the job of the signs of alternating rows, for the case when the sequence changes.
\end{abstract} functions.

Key words: row, n-th member, alternating series, sneakerboy series, trigonometric

Citation: Shevtsov AN, Yeskali NZ, Sabdenbek AA, Uakbayev MM (2014) ABOUT THE PROBLEM OF ARBITRARY ALTERNATING PLUS AND MINUS SIGNS IN ARITHMETIC SERIES. ISJ Theoretical \& Applied Science 9 (17): 5-22. doi: http://dx.doi.org/10.15863/TAS.2014.09.17.2

\section{О ПРОБЛЕМЕ ПРОИЗВОЛЬНОГО ЧЕРЕДОВАНИЯ ЗНАКОВ ПЛЮС И МИНУС В АРИФМЕТИЧЕСКИХ РЯДАХ}

Аннотация: При нахождении суммы и п-го члена знакопеременного ряда возникает ряд проблем когда ряд не является знакочередующимся. В статье приводятся несколько формул, для решения данной проблемы задания знаков знакопеременных рядов, для случаякогда последовательность знаков меняется.

Ключевые слова: ряд, п-й член, знакопеременный ряд, знакочередующийся ряд, тригонометрические функиии.

\section{ВВЕДЕНИЕ}

При изучении арифметических знакопеременных рядов [1-4], ряды 2577, 2660, 2662, 2666, 2694, 2695, приводятся без $n$-го члена ряда [1, с. 250, 260-262], а также и в других источниках, встречаются задачи где аналогично $n$-й член ряда просто опускается. Это 
происходит из-за сложности восприятия, и отсутствия аналитических формул, когда последовательность знаков начинает отличаться от привычного значения $\sum_{n=1}^{\infty}(-1)^{n+1} u_{n}$ :

$$
+-+-+-+-+-+-+-\ldots . .
$$

В указанных задачах встречается только несколько вариантов:

$$
++-++-++-++-++-\ldots \ldots . . .
$$

И

$$
+--+--+--+--+--+--\ldots \ldots
$$

А также встречается и такой вариант:

$$
+++---+++---+++---\ldots \ldots
$$

Хотя, конечно же очевидно, что таких вариантов намного больше, и построение $n$ го члена ряда будет напрямую зависеть от порядка, количества и периодичности чередования знаков. Например, задавая некоторую функцию, мы получим последовательность из двух минусов и остальных плюсов (Рис.1).

$$
\sum_{n=0}^{\infty}\left(\frac{n^{2}-8}{\left|n^{2}-8\right|}\right) u_{n} .
$$

restart;

$\mathrm{r}:=\mathrm{n}^{\wedge} 2-8$;

$\mathrm{plot}(\mathrm{r}, \mathrm{n}=0.10)$;

$$
r:=n^{2}-8
$$

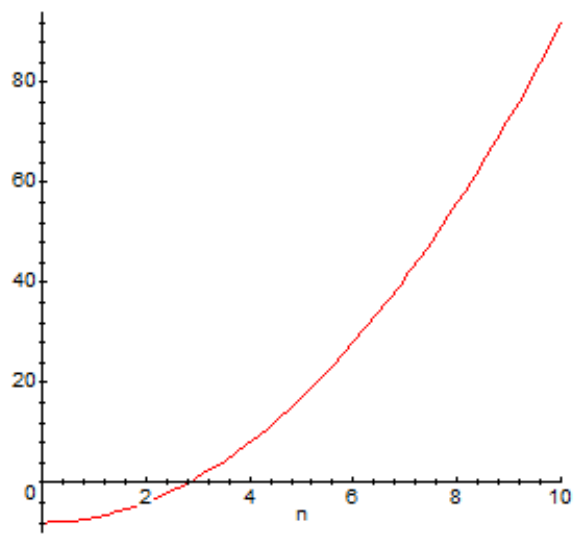

Рисунок 1 - Пример последовательности ряда (1).

\section{АЛГОРИТМЫ И МЕТОДЫ РЕШЕНИЯ}

Для решения данной задачи, будем определять аналитическое решение. Найдем $n$ й член ряда, с учетом знака функции (1). При этом, $n$-й член ряда можно будет определить как 


$$
\sum_{n=0}^{\infty}\left(\frac{n^{2}-\left(b+\frac{1}{2}\right)^{2}}{\left|n^{2}-\left(b+\frac{1}{2}\right)^{2}\right|}\right) u_{n}
$$

где $b$ - это количество первых отрицательных слагаемых арифметического ряда. В данном ряде (1), для изменения знаков на противоположные, необходимо умножить ряд на минус единицу:

т.е. получим

$$
--+++++++++++++\ldots .
$$

$$
\sum_{n=0}^{\infty}\left(-1^{b}\right)^{a}\left(\frac{n^{2}-\left(a+b+\frac{1}{2}\right)^{2}}{\left|n^{2}-\left(a+b+\frac{1}{2}\right)^{2}\right|} u_{n},\right.
$$

где $a$ - количество первых положительных слагаемы арифметического ряда.

Введем новую функцию

$$
\tilde{r}(r)=\frac{r}{|r|}, \quad r>0
$$

Теперь (3) с учетом (4) примет вид:

$$
\sum_{n=0}^{\infty}\left(-1^{b}\right)^{a}\left(\tilde{r}\left(n^{2}-\left(a+b+\frac{1}{2}\right)^{2}\right)\right) u_{n} .
$$

При этом следует учесть, что последующие слагаемые не учитываются, а в расчет берутся только первые слагаемые ряда:

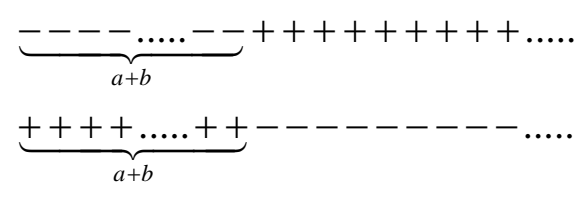

Рассмотрим теперь периодический случай:

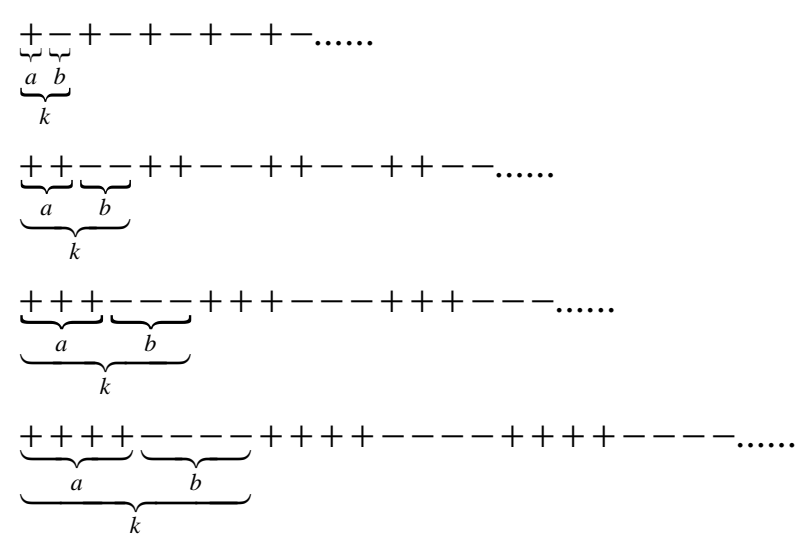


$n$-й член ряда можно записать в виде

$$
\sum_{n=1}^{\infty} \tilde{r}\left(\sin \left(\frac{2 \pi n}{k}\right)\right) u_{n}=\sum_{n=1}^{\infty} \tilde{r}\left(\sin \left(\frac{\pi n}{\left(\frac{a+b}{2}\right)}\right)\right) u_{n} .
$$

Или аналогично

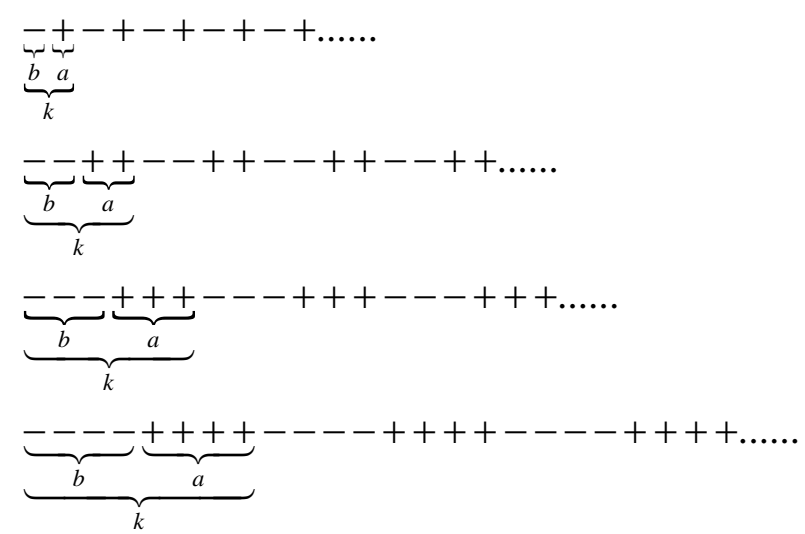

Тогда, $n$-й член ряда получим в следующем виде

$$
\sum_{n=1}^{\infty} \tilde{r}\left(\sin \left(\frac{2 \pi n}{k}\right)\right) u_{n}=\sum_{n=1}^{\infty} \tilde{r}\left(\sin \left(\frac{\pi n}{\left(\frac{a+b}{2}\right)}\right)\right) u_{n} .
$$

Сделаем сдвиг на $\frac{1}{2}$ от шага в 1 , тогда получим:

$$
\sum_{n=1}^{\infty} \tilde{r}\left(\sin \left(\frac{\pi\left(n-\frac{1}{2}\right)}{\left(\frac{a+b}{2}\right)}\right)\right) u_{n} .
$$

Разработаем алгоритмы и программу на Delphi Architect для расчета значений полученной функции (8). 


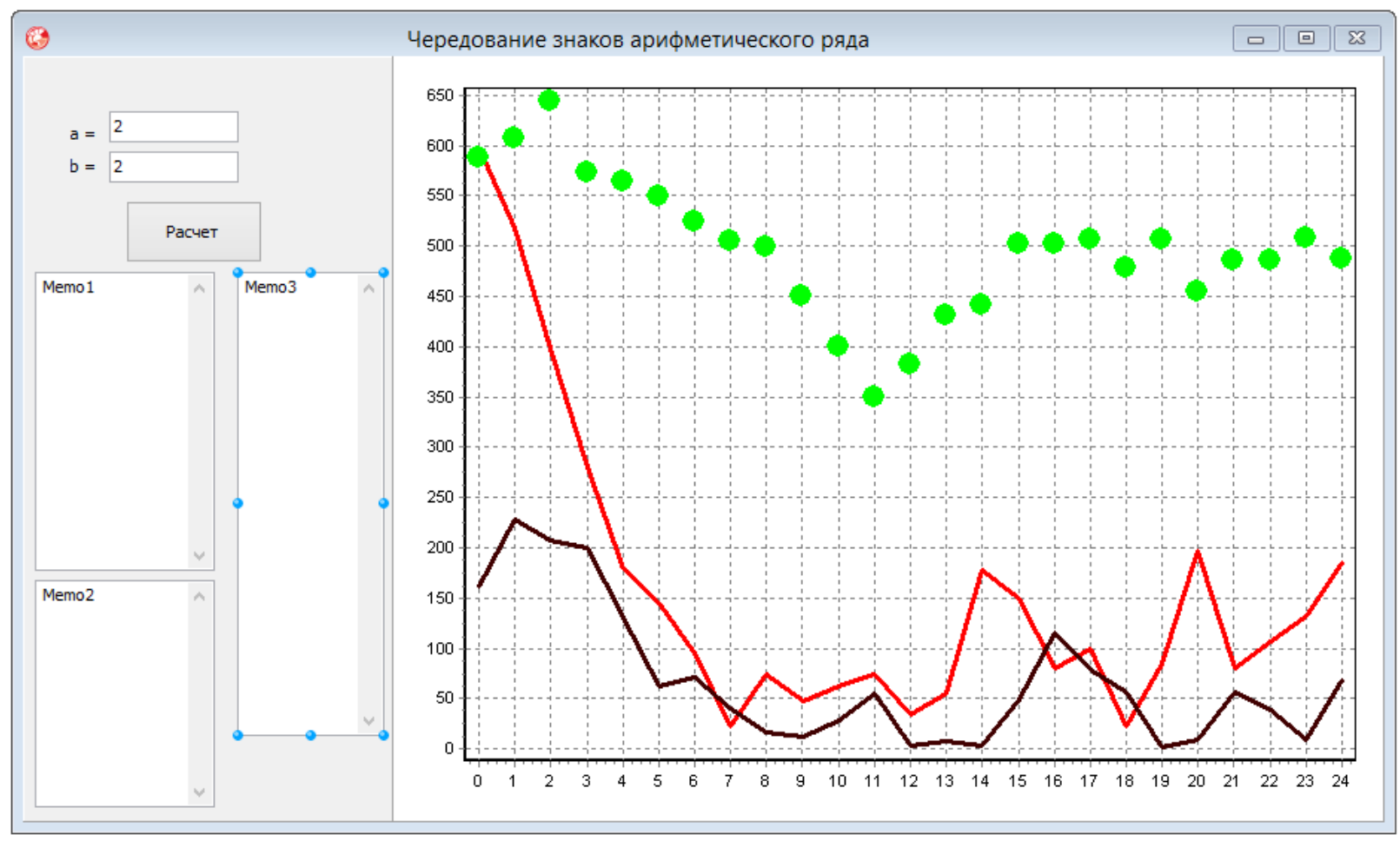

Рисунок 2 - Разработка программы.

unit Unit1;

interface

uses

Winapi.Windows, Winapi.Messages, System.SysUtils, System.Variants, System.Classes, Vcl.Graphics,

Vcl.Controls, Vcl.Forms, Vcl.Dialogs, Vcl.StdCtrls, VCLTee.TeEngine,

VCLTee.Series, VCLTee.TeeProcs, VCLTee.Chart, Vcl.ExtCtrls;

type

TForm1 $=$ class $($ TForm $)$

Panel1: TPanel;

Chart1: TChart;

Series1: TLineSeries;

Label1: TLabel;

Edit1: TEdit;

Label2: TLabel;

Edit2: TEdit;

Memo1: TMemo;

Memo2: TMemo;

Series2: TLineSeries;

Button1: TButton;

Memo3: TMemo;

Series3: TPointSeries;

procedure Button1Click(Sender: TObject);

private

\{ Private declarations \}

public

\{ Public declarations \} 


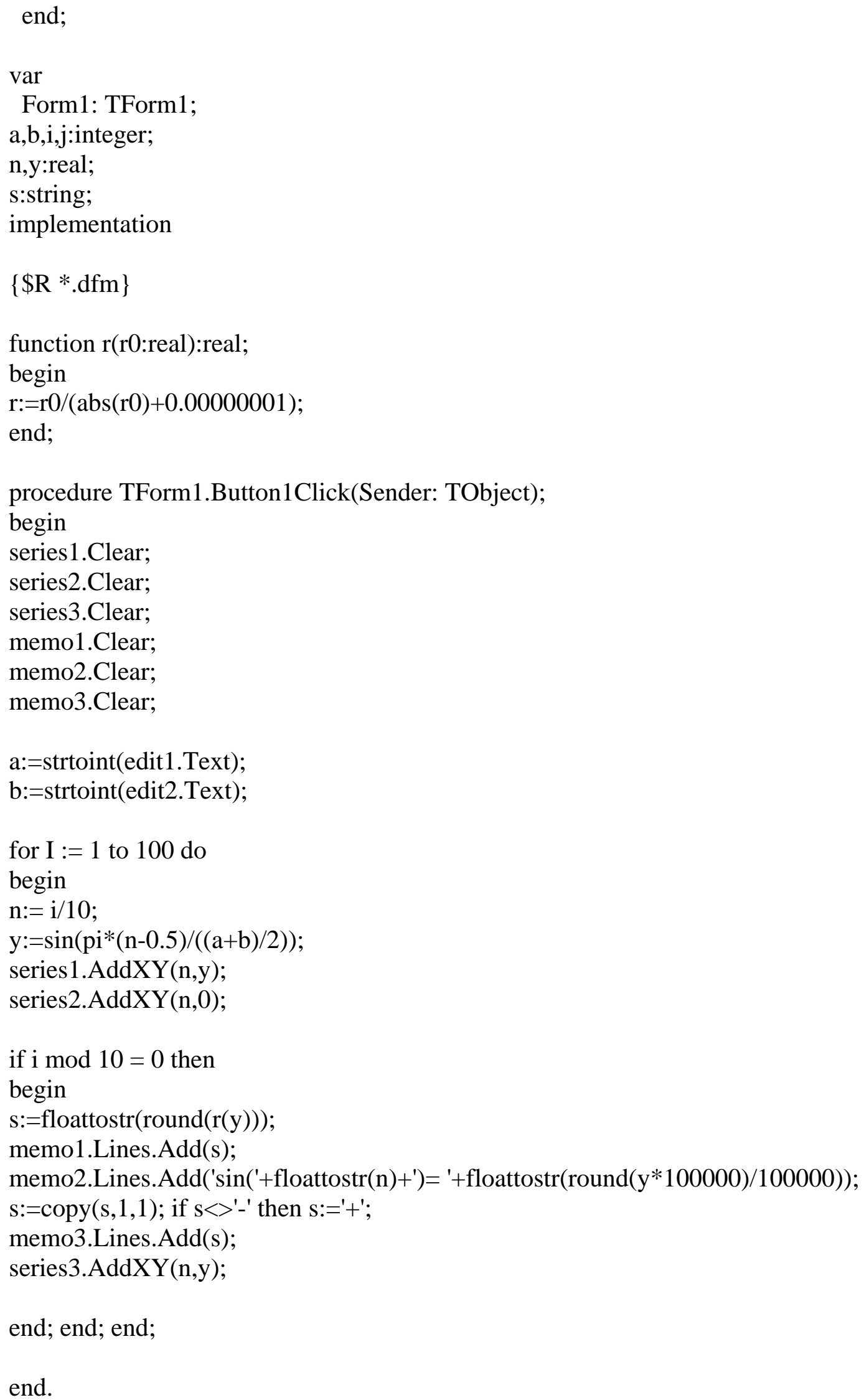

Осуществим расчет значений функции $\tilde{r}$, для случая $a=b$, при $a=1 \div 10$. 


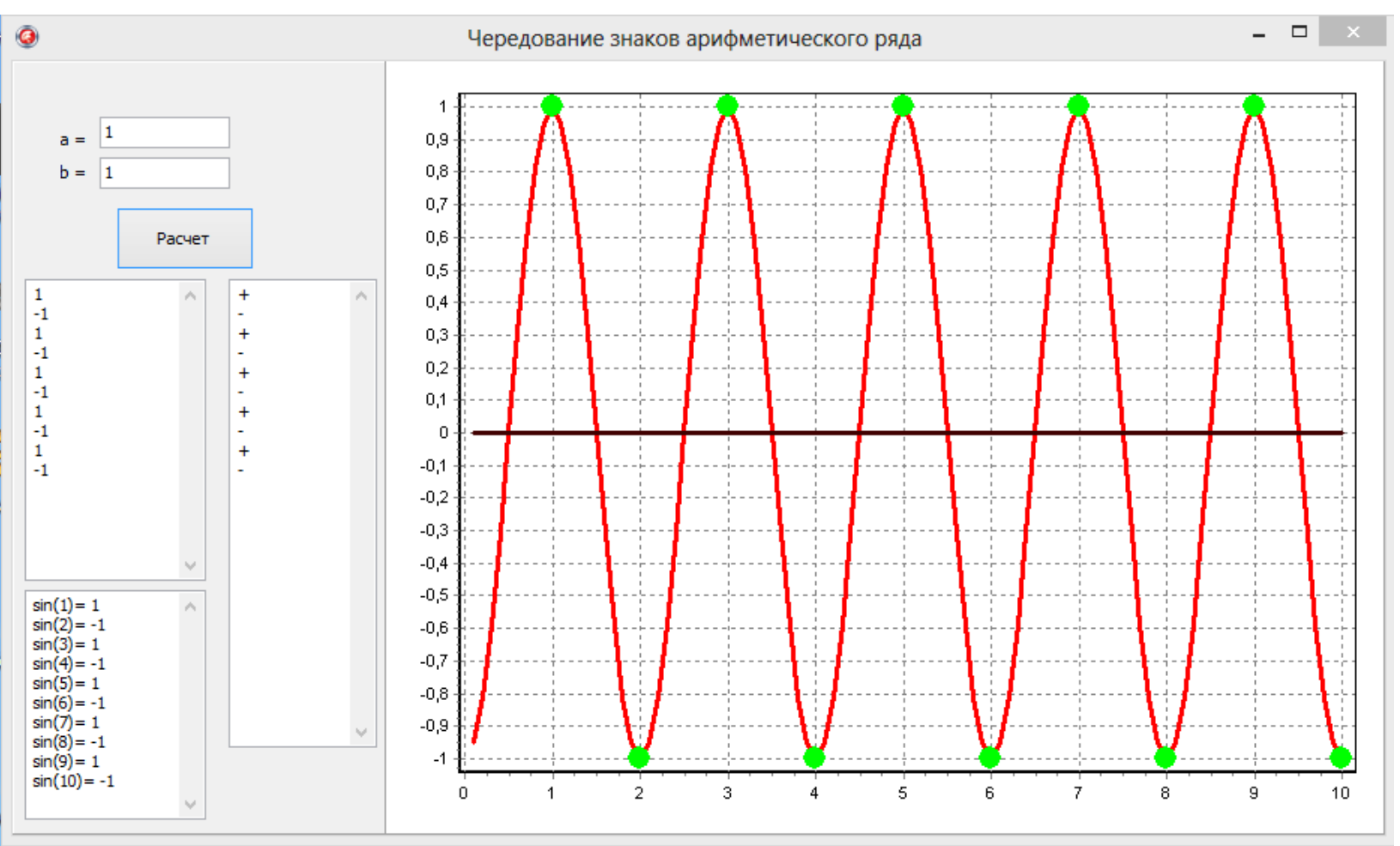

Рисунок 3 - Расчет значений функции $\tilde{r}$, для случая $a=b=1$.

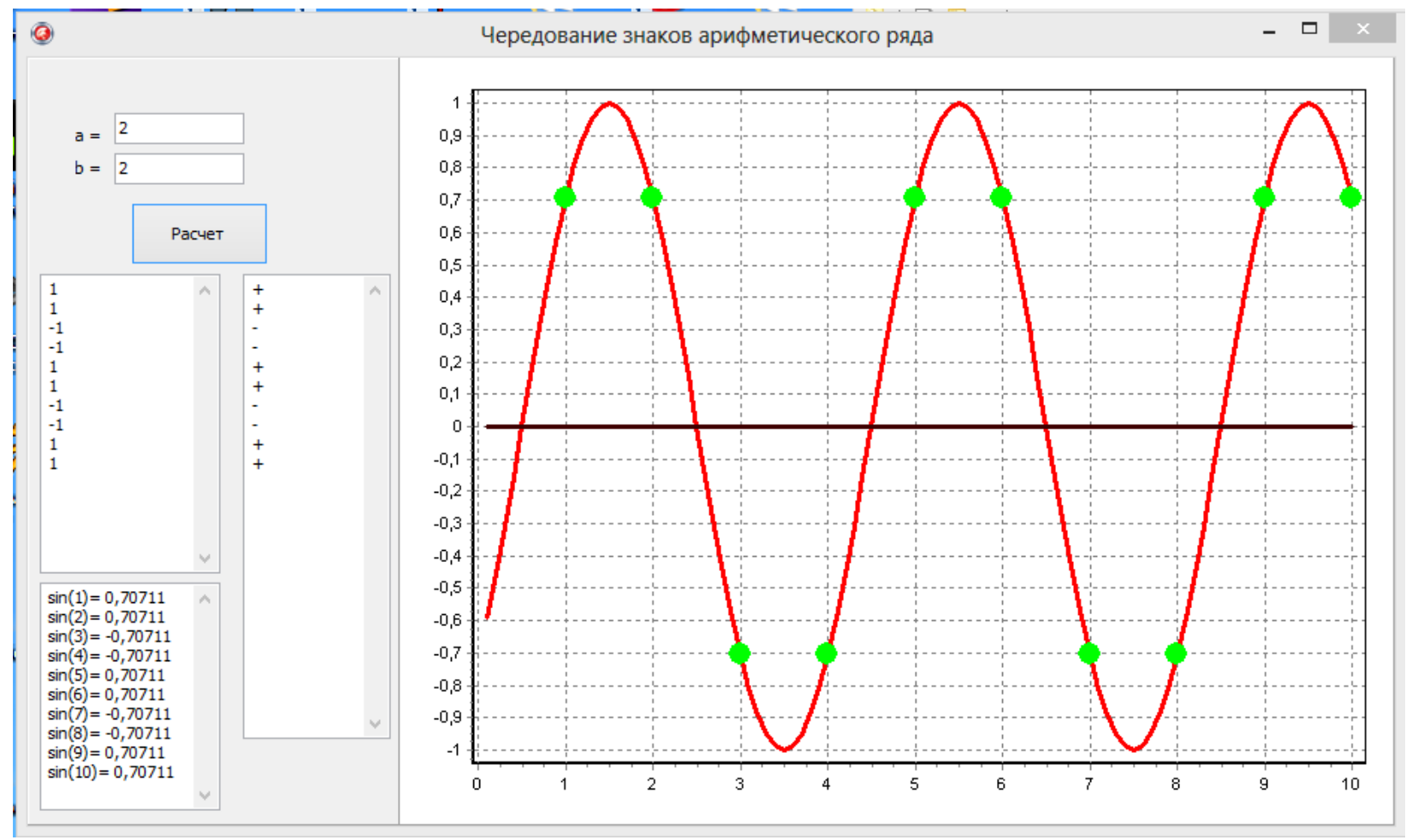

Рисунок 4 - Расчет значений функции $\tilde{r}$, для случая $a=b=2$. 


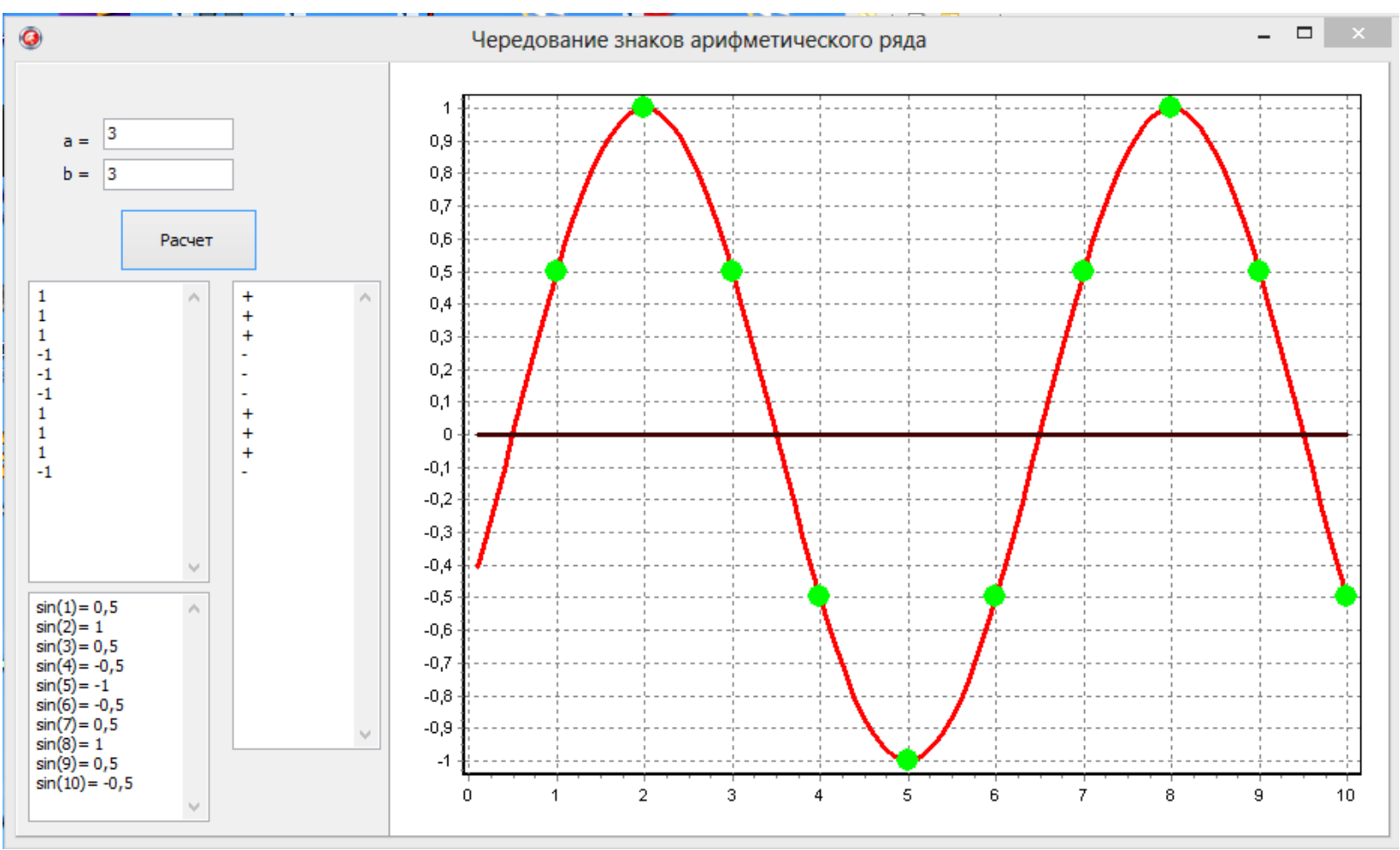

Рисунок 5 - Расчет значений функции $\tilde{r}$, для случая $a=b=3$.

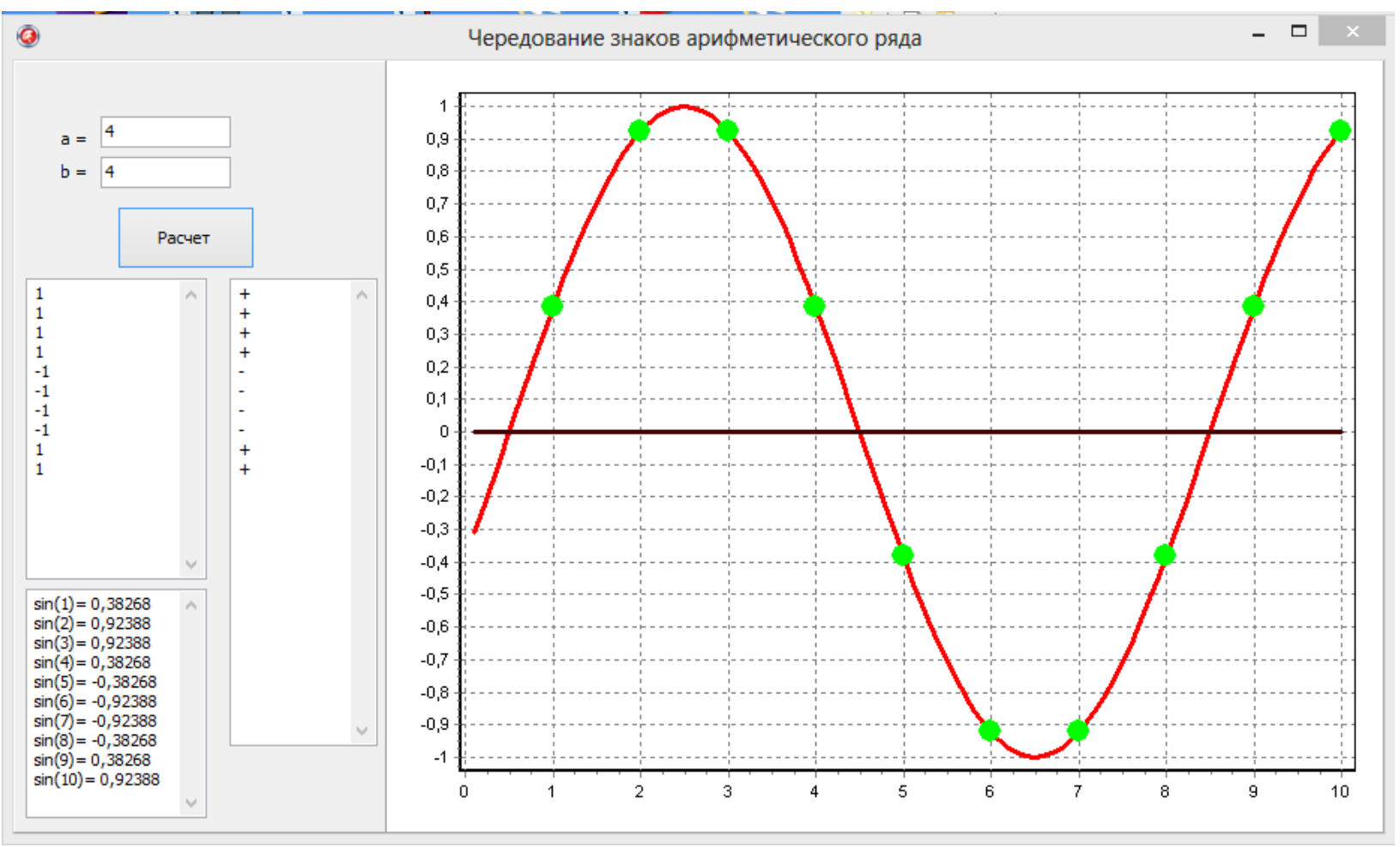

Рисунок 6 - Расчет значений функции $\tilde{r}$, для случая $a=b=4$. 


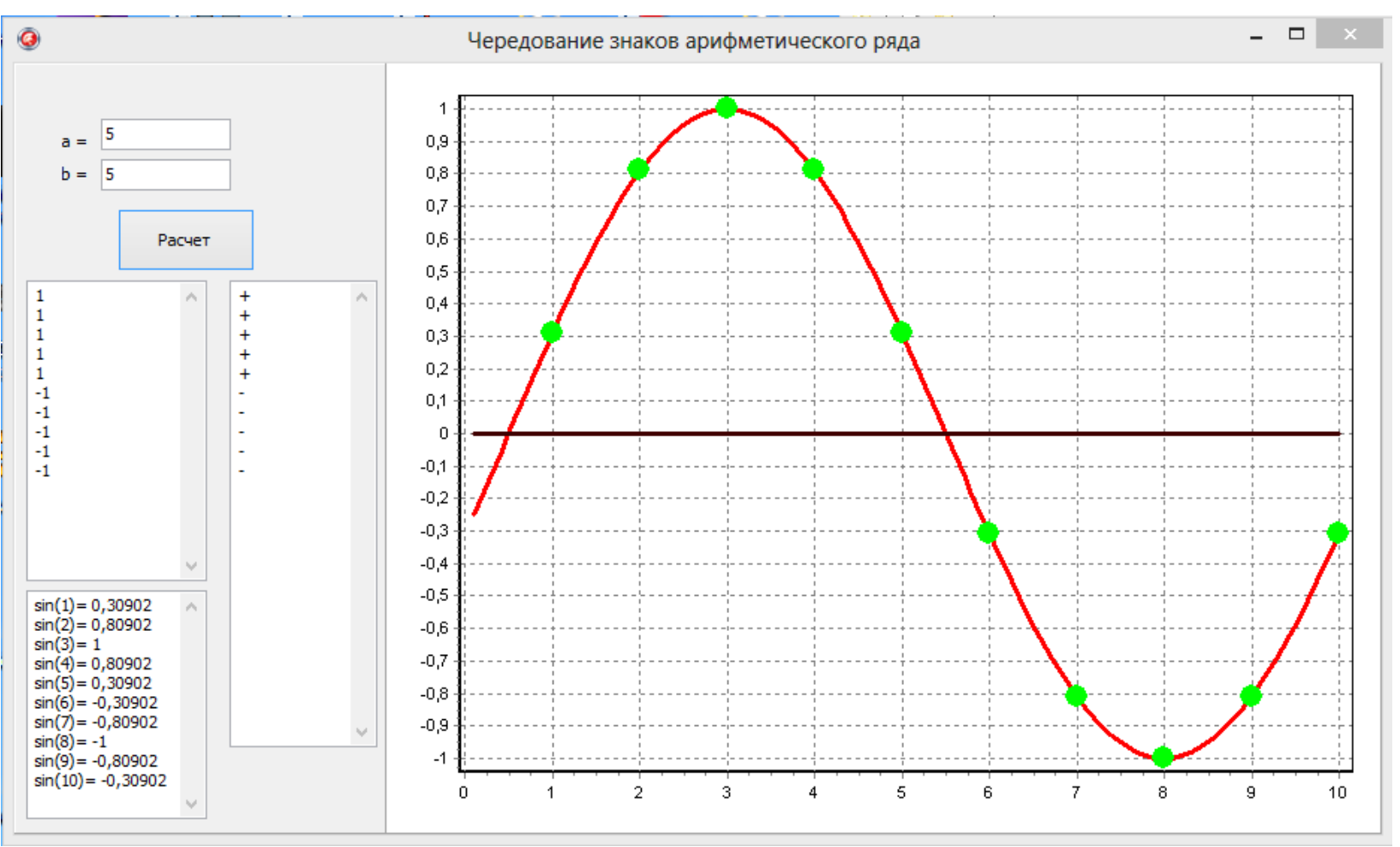

Рисунок 7 - Расчет значений функции $\tilde{r}$, для случая $a=b=5$.

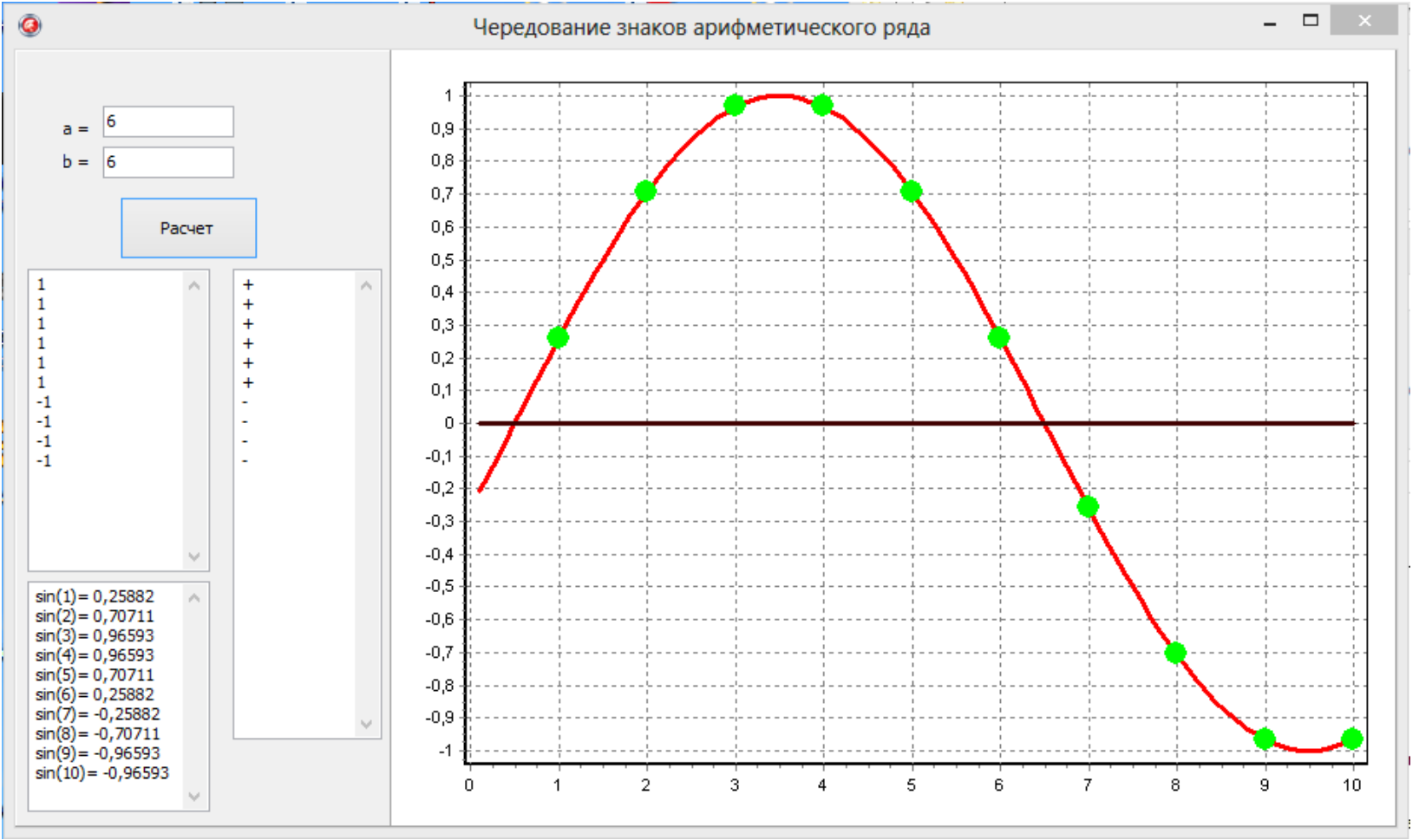

Рисунок 8 - Расчет значений функции $\tilde{r}$, для случая $a=b=6$. 


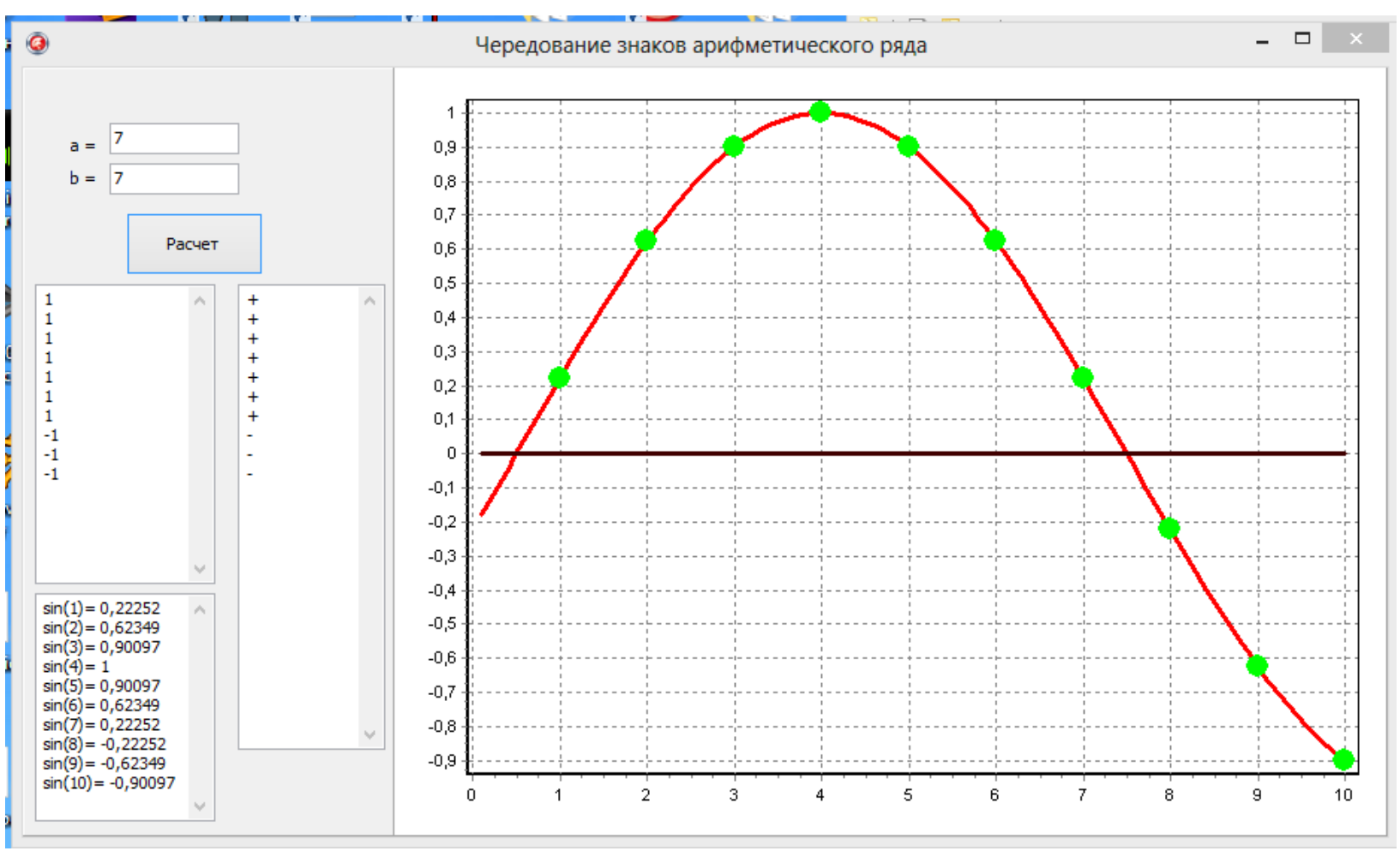

Рисунок 9 - Расчет значений функции $\tilde{r}$, для случая $a=b=7$.

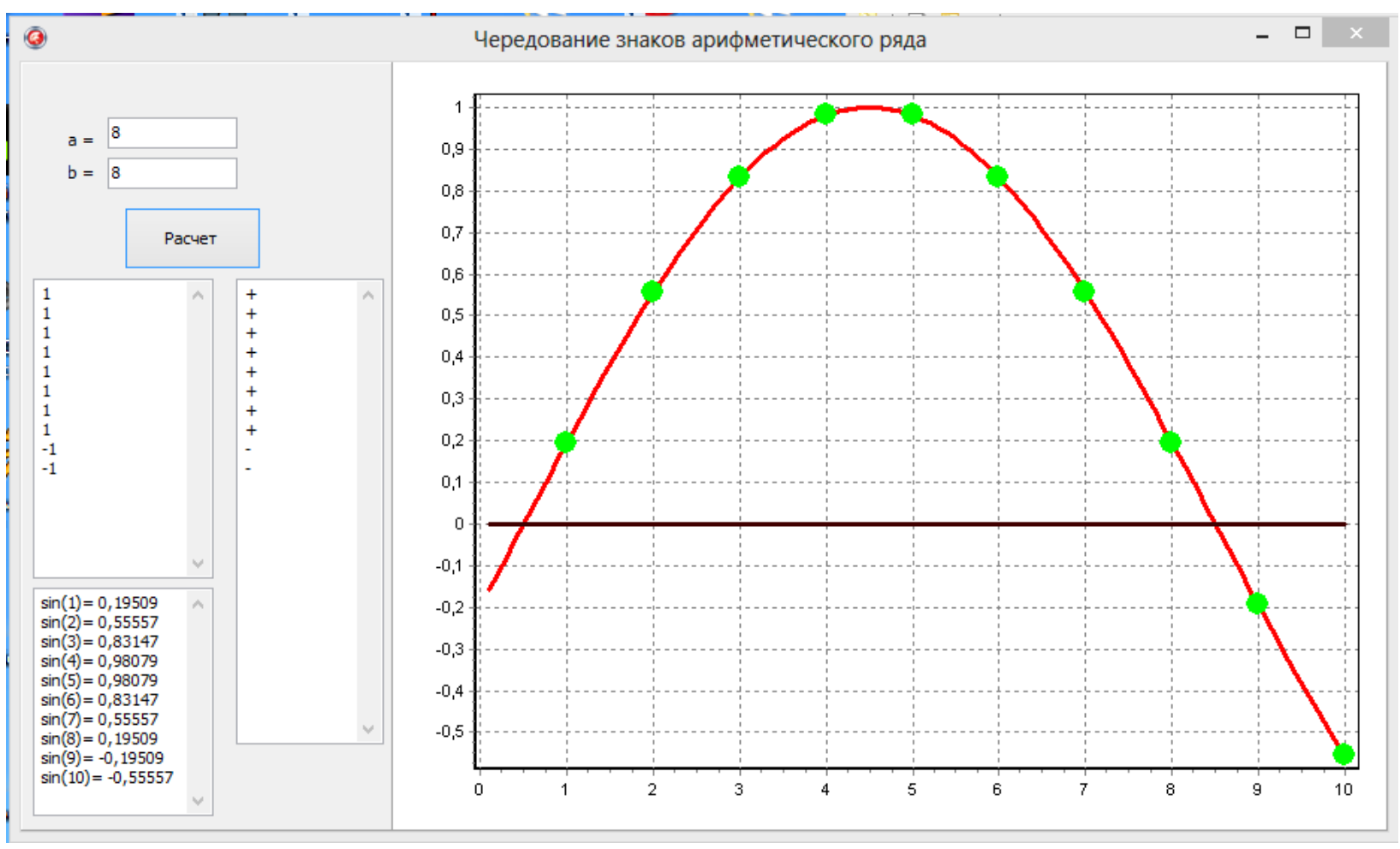

Рисунок 10 - Расчет значений функции $\tilde{r}$, для случая $a=b=8$. 


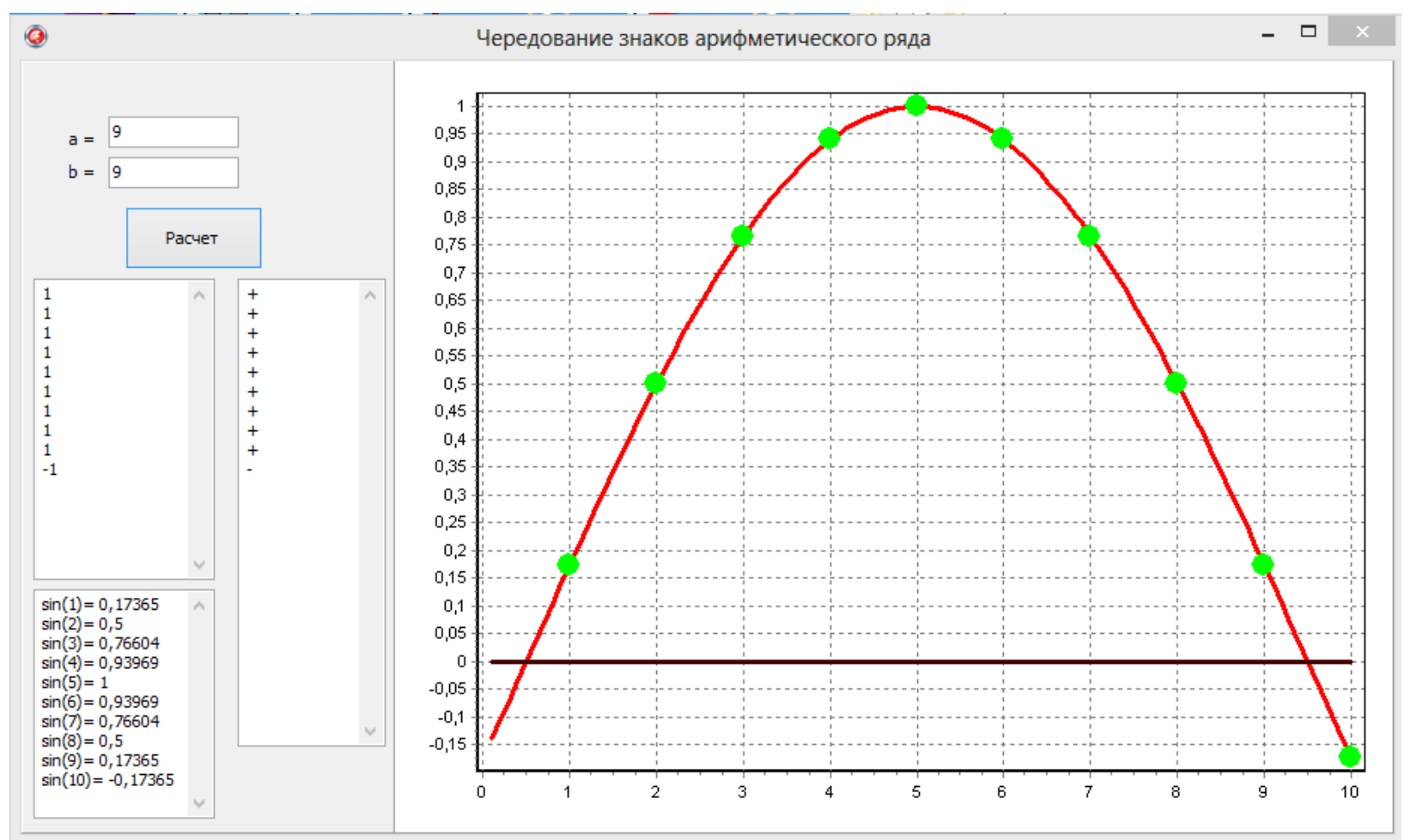

Рисунок 11 - Расчет значений функции $\tilde{r}$, для случая $a=b=9$.

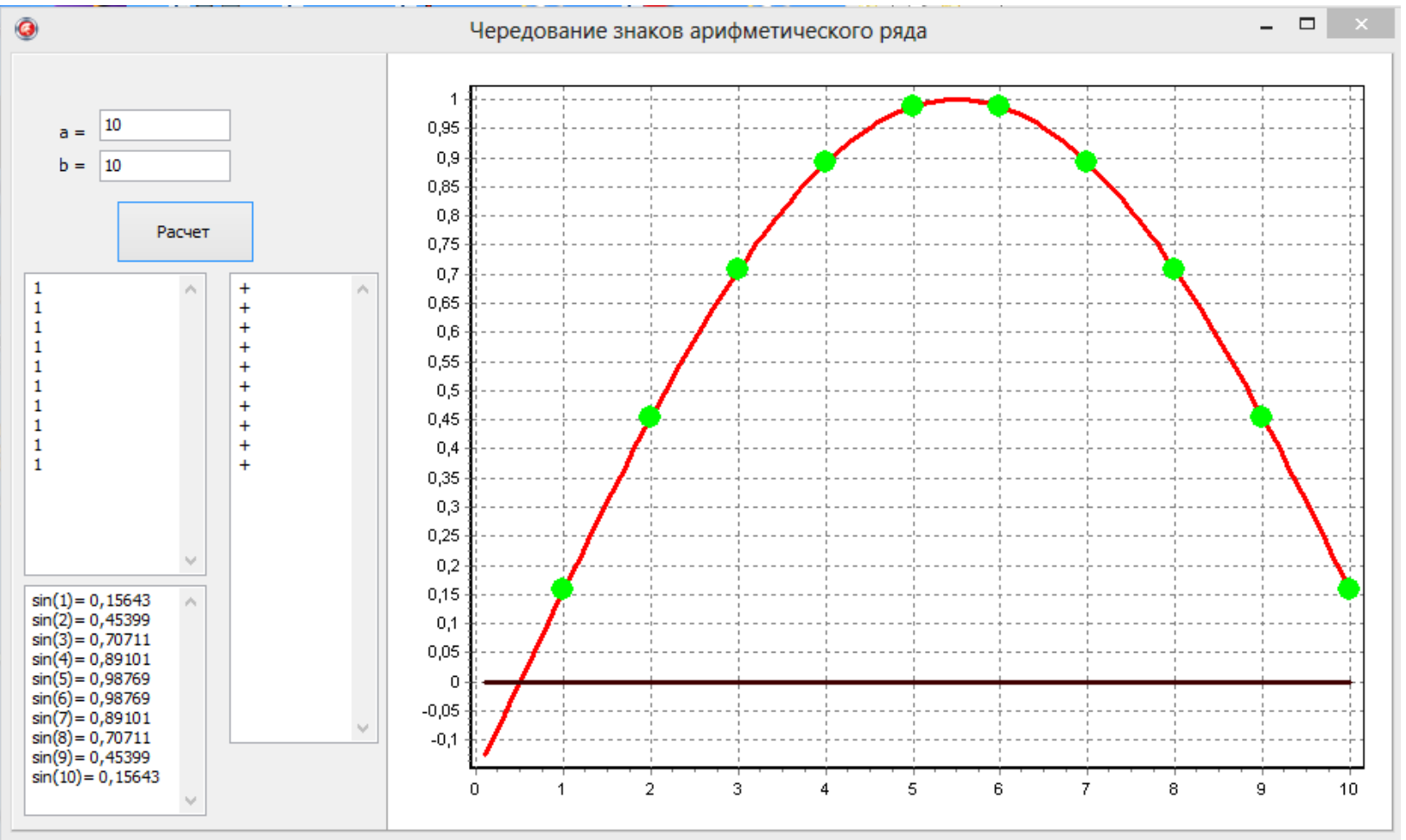

Рисунок 12 - Расчет значений функции $\tilde{r}$, для случая $a=b=10$.

Отдельно хочется отметить, что для некоторых значений $a$ и $b$, когда $a>b-$ полученная формула (8) остается актуальной. 


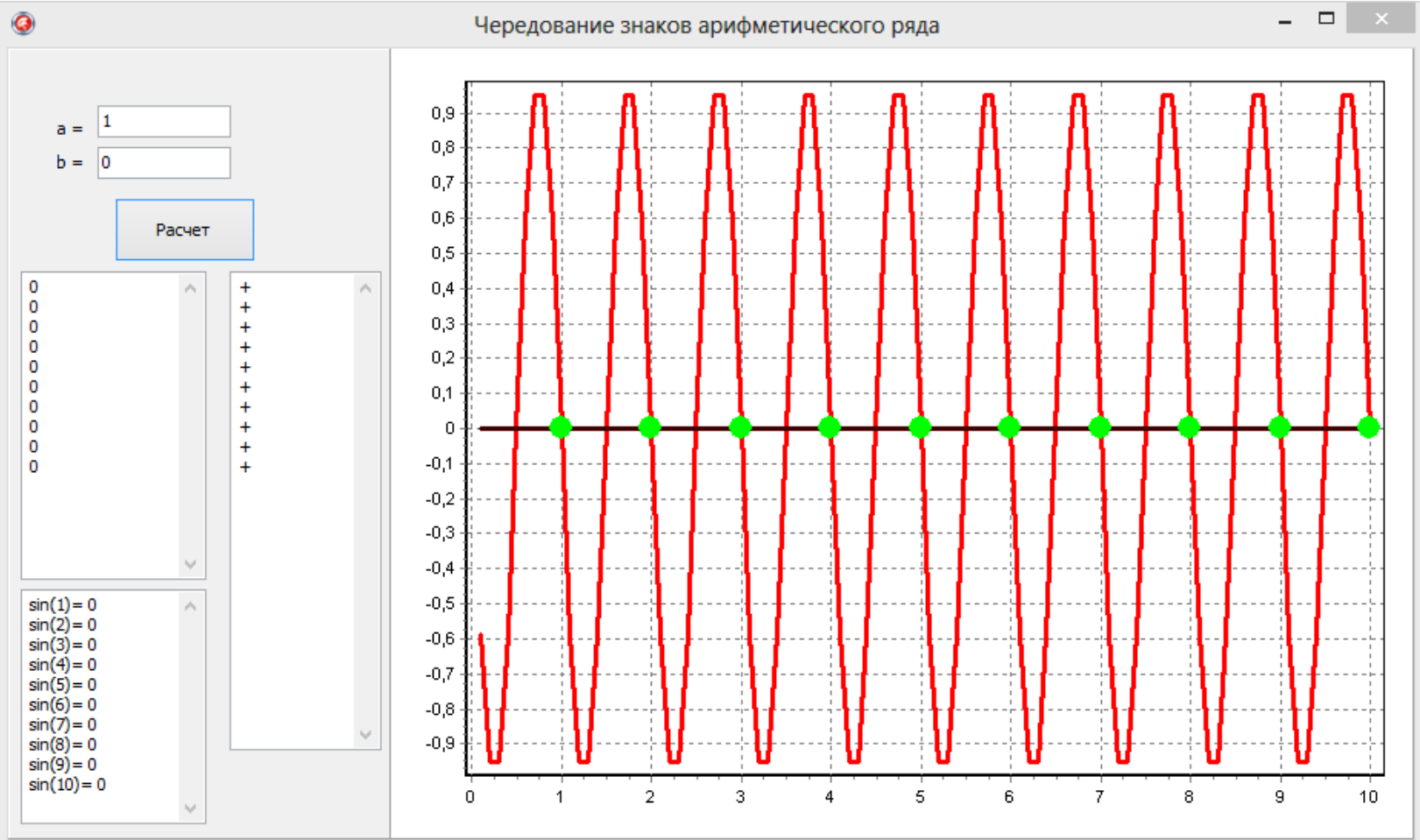

Рисунок 13 - Расчет значений функции $\tilde{r}$, для случая $a=1, b=0$.

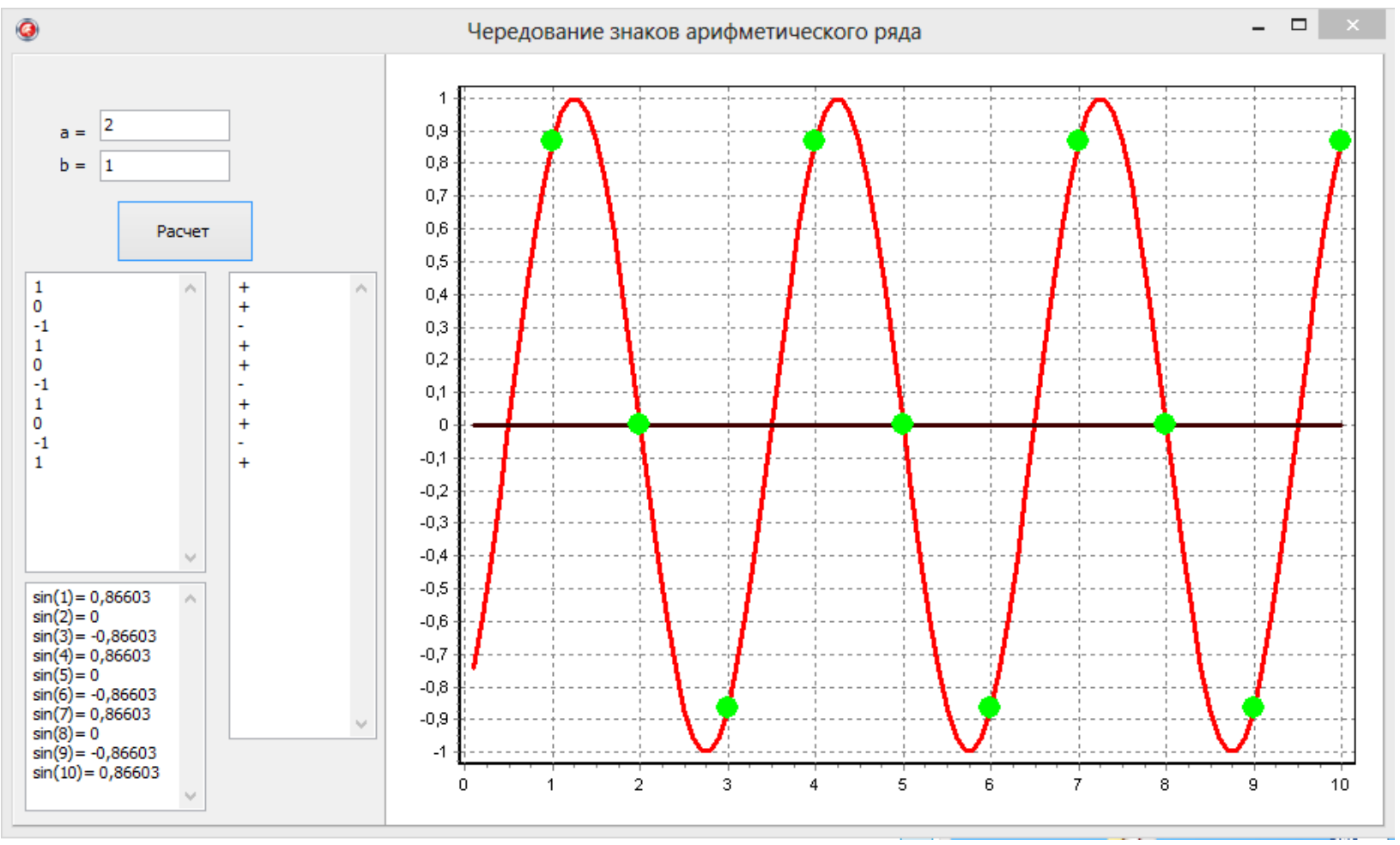

Рисунок 14 - Расчет значений функции $\tilde{r}$, для случая $a=2, b=1$. 


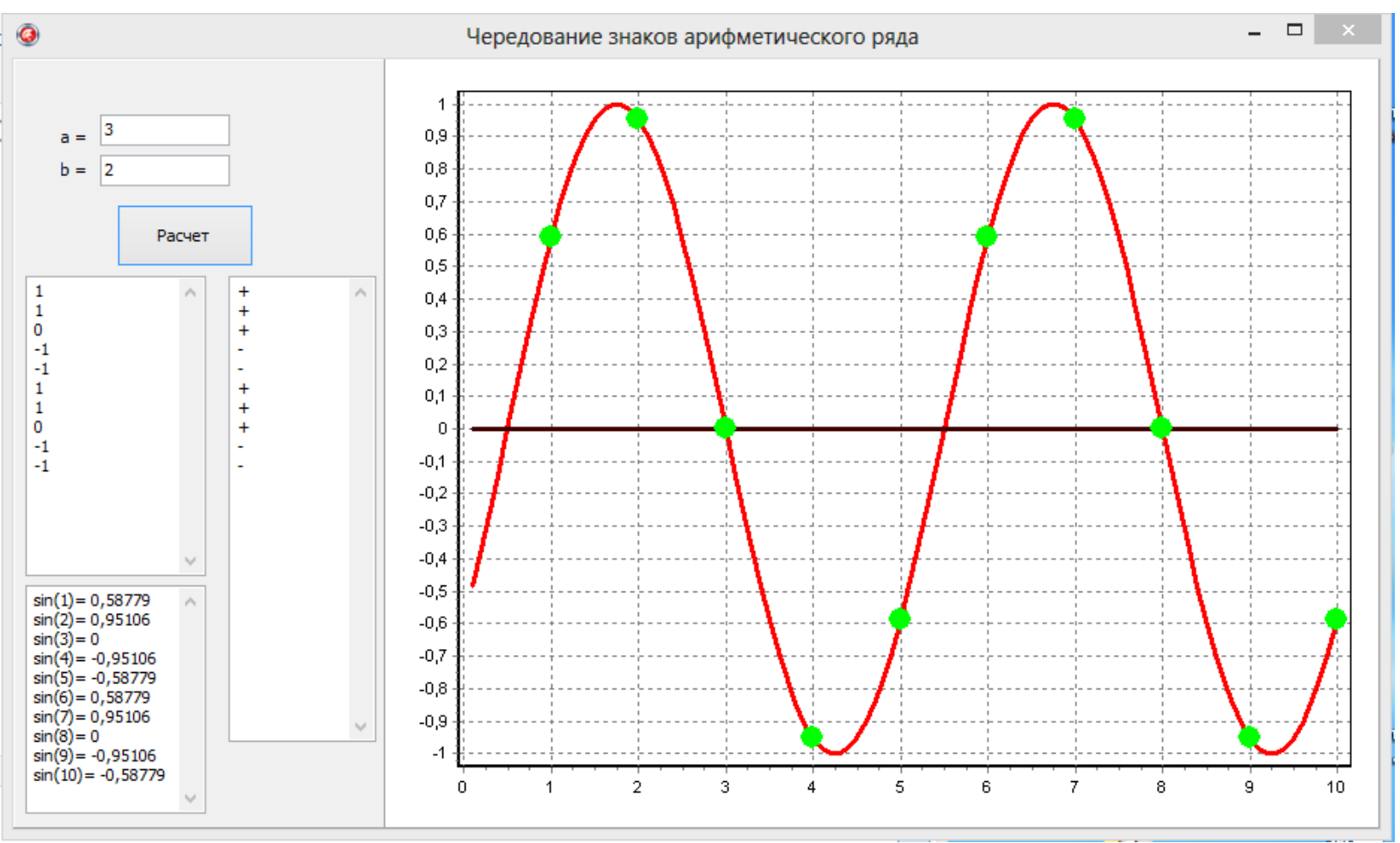

Рисунок 15 - Расчет значений функции $\tilde{r}$, для случая $a=3, b=2$.

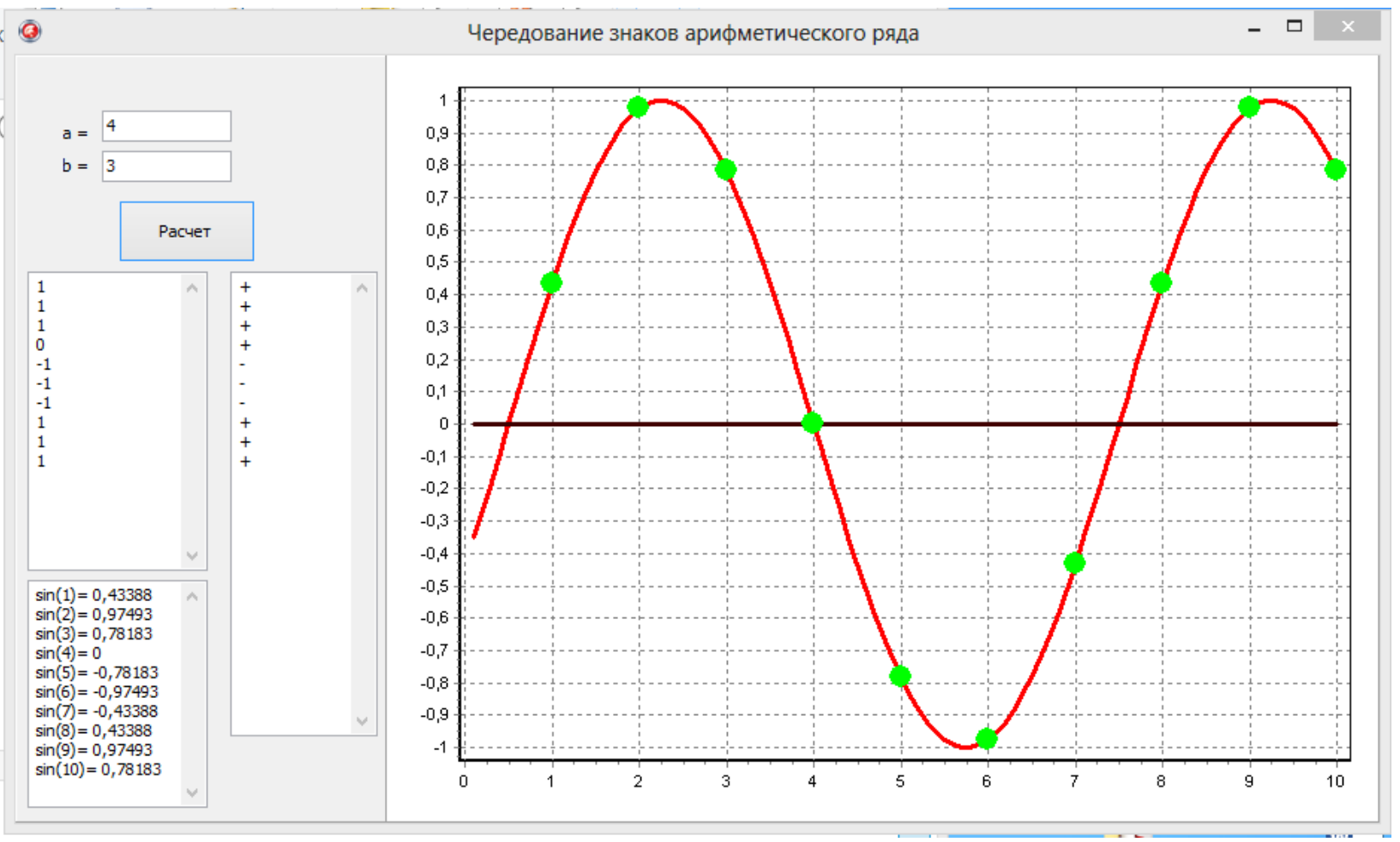

Рисунок 16 - Расчет значений функции $\tilde{r}$, для случая $a=4, b=3$. 


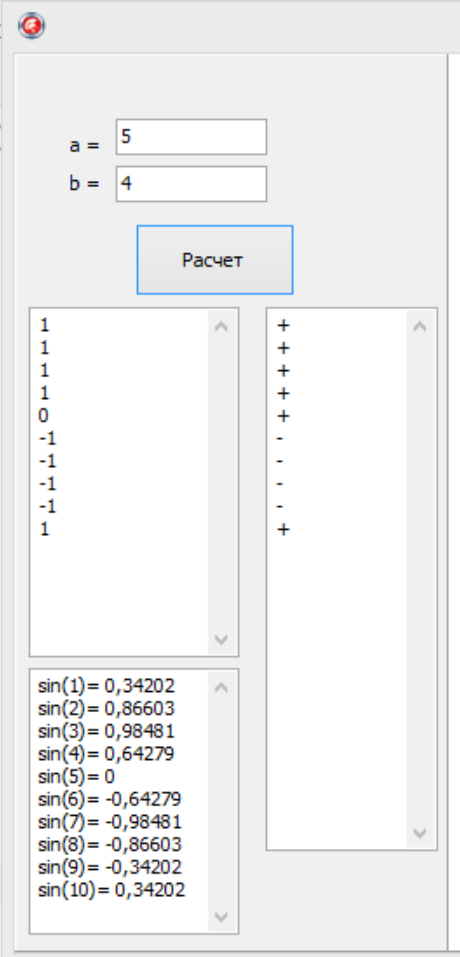

Чередование знаков арифметического ряда

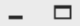

Рисунок 17 - Расчет значений функции $\tilde{r}$, для случая $a=5, b=4$.
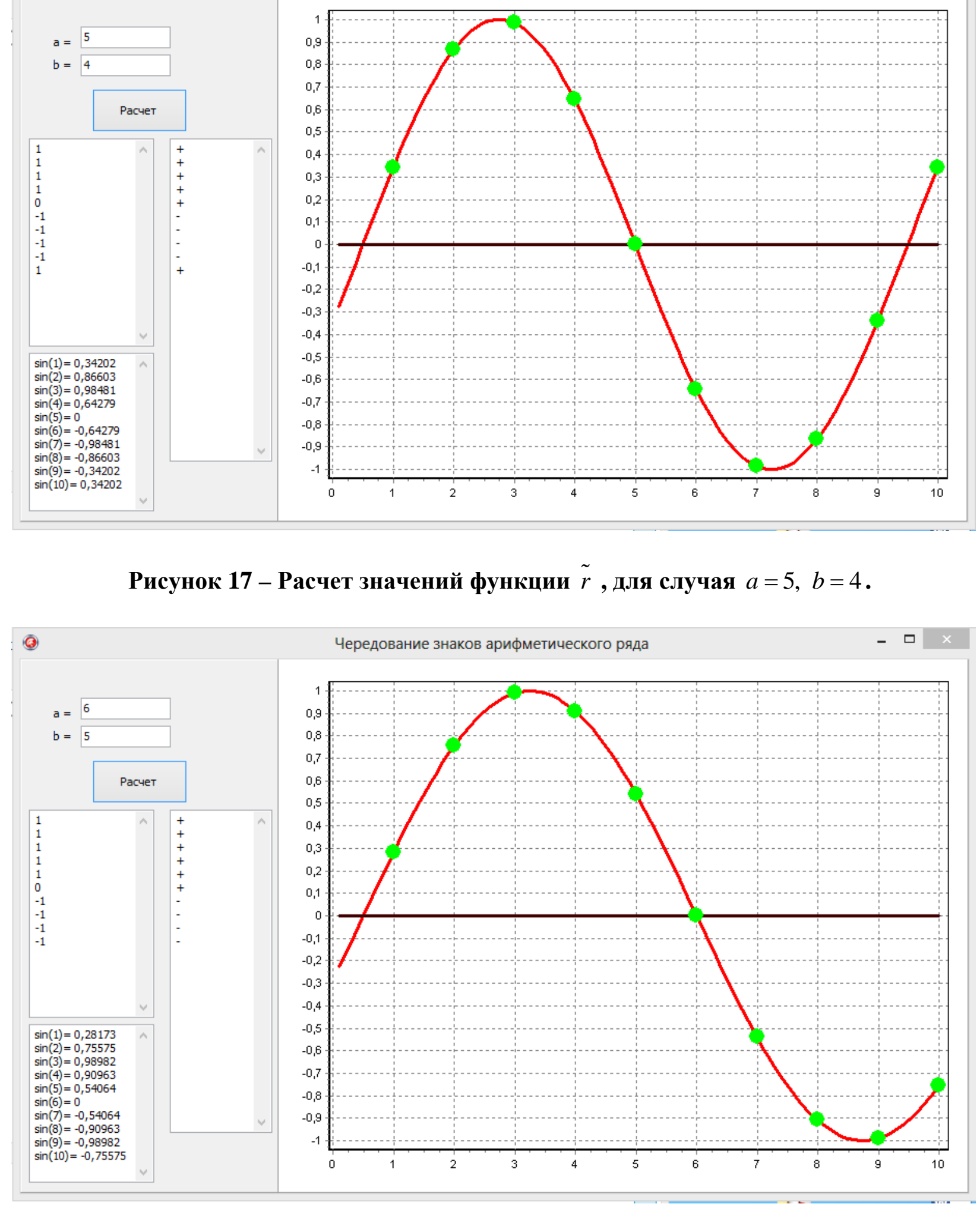

Рисунок 18 - Расчет значений функции $\tilde{r}$, для случая $a=6, b=5$. 


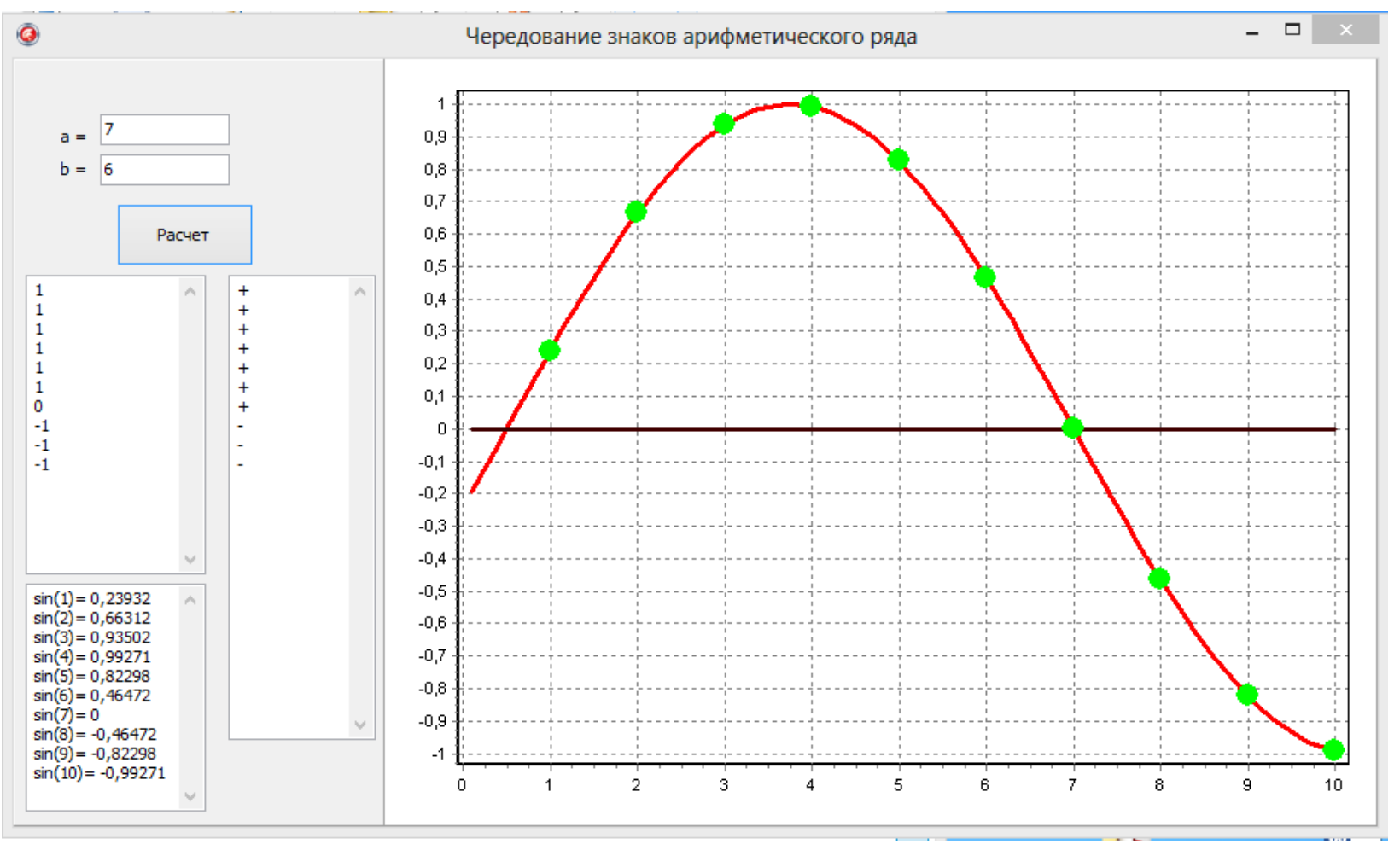

Рисунок 19 - Расчет значений функции $\tilde{r}$, для случая $a=7, b=6$.
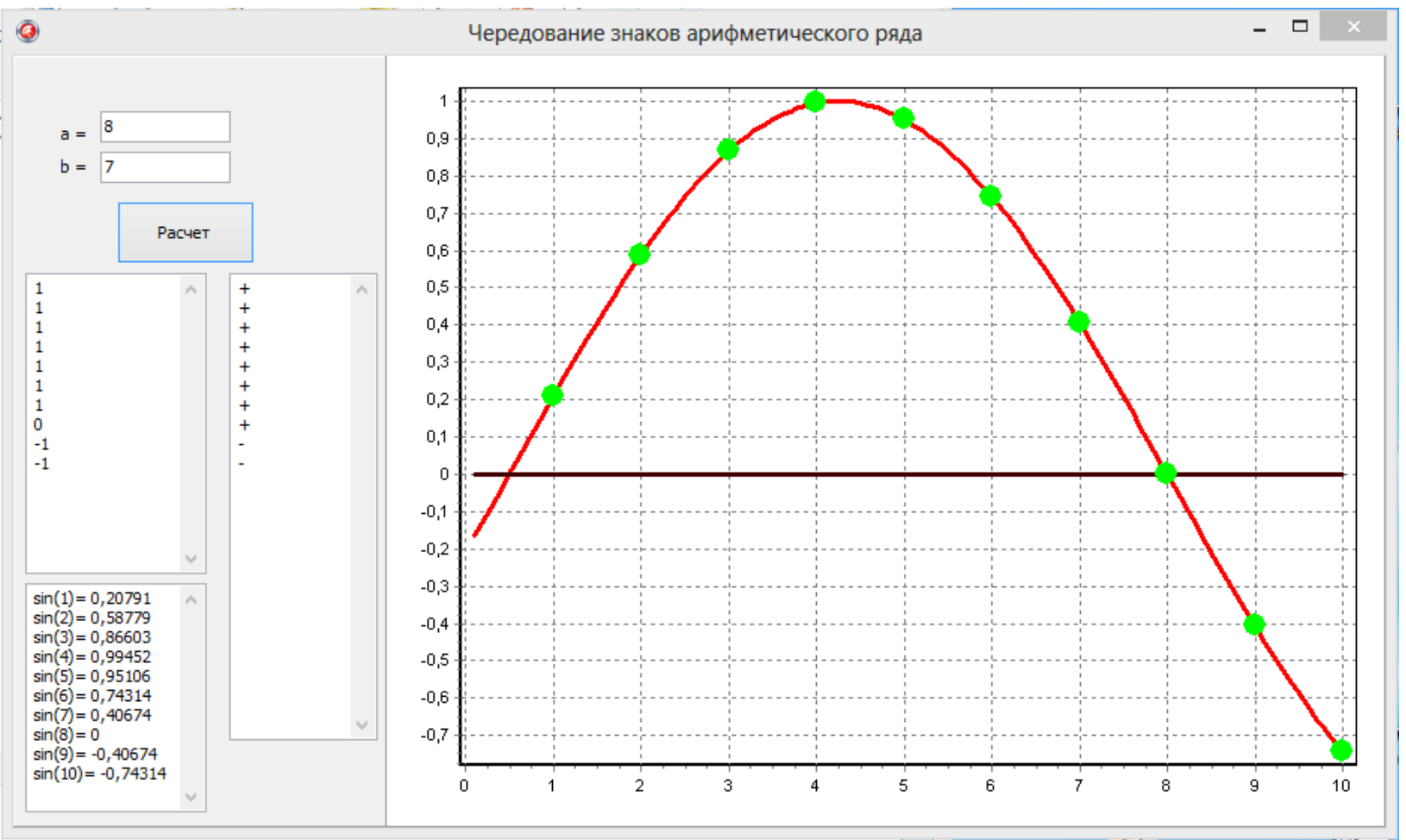

Рисунок 20 - Расчет значений функции $\tilde{r}$, для случая $a=8, b=7$. 


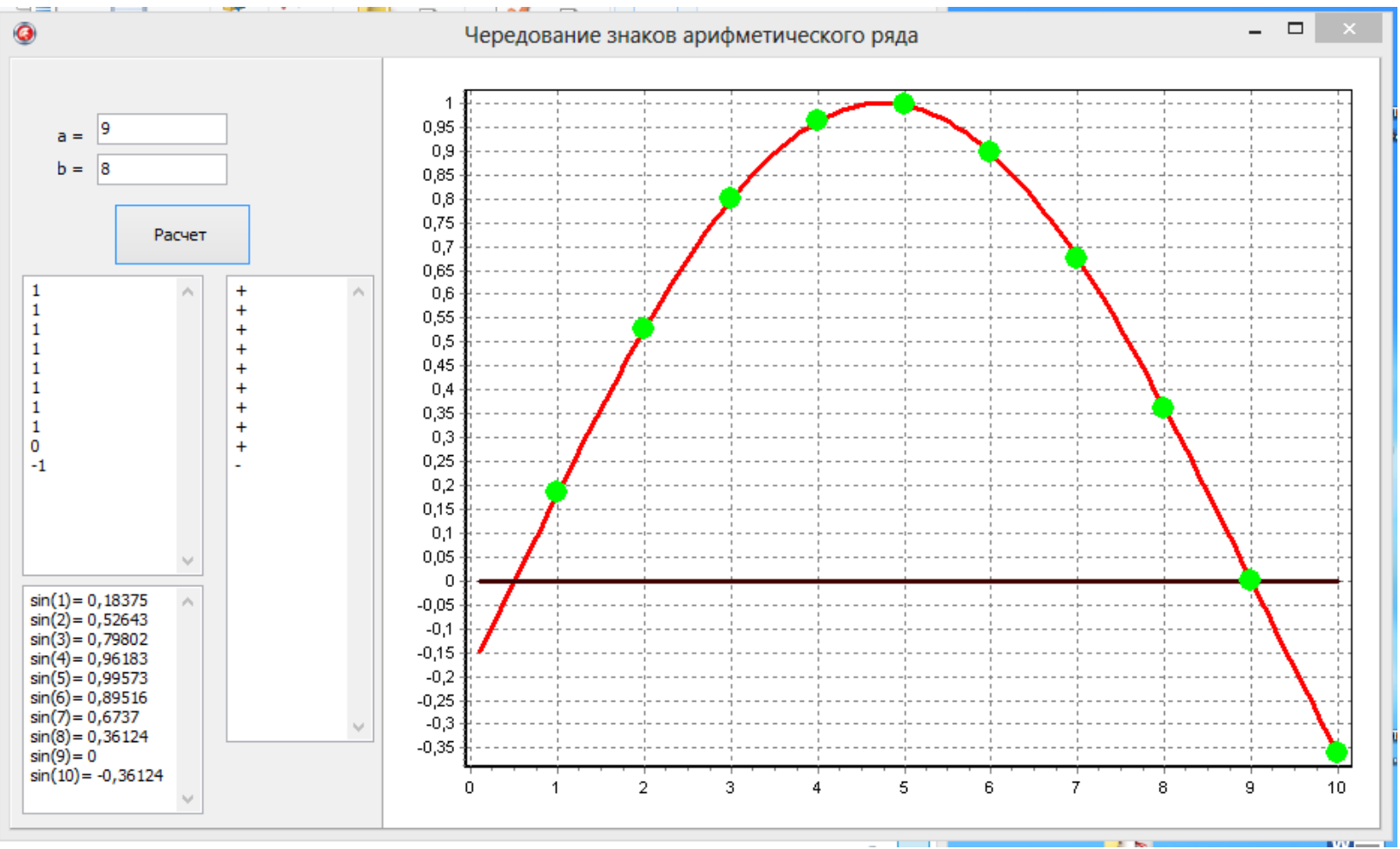

Рисунок 21 - Расчет значений функции $\tilde{r}$, для случая $a=9, b=8$.

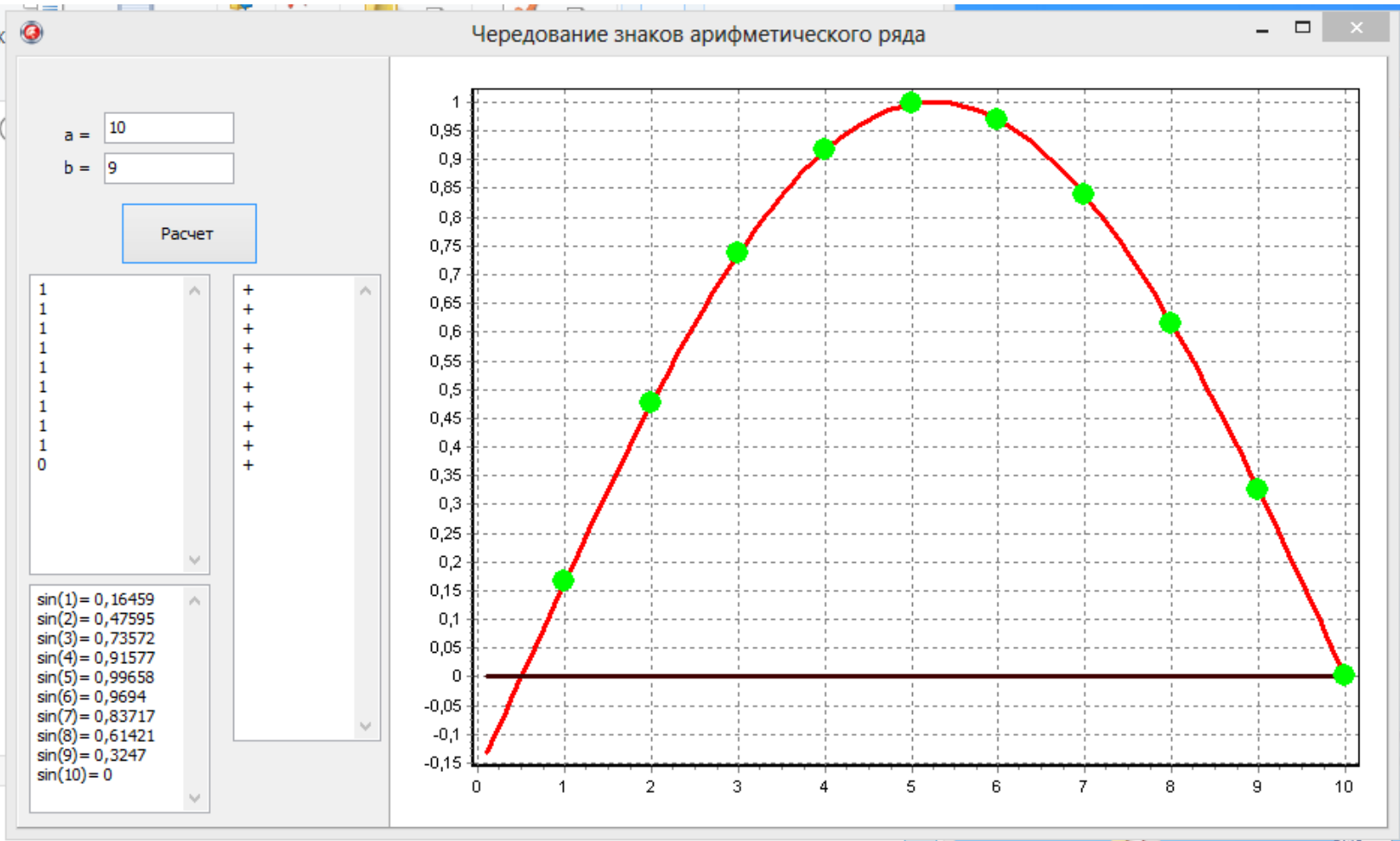

Рисунок 22 - Расчет значений функции $\tilde{r}$, для случая $a=10, b=9$. 


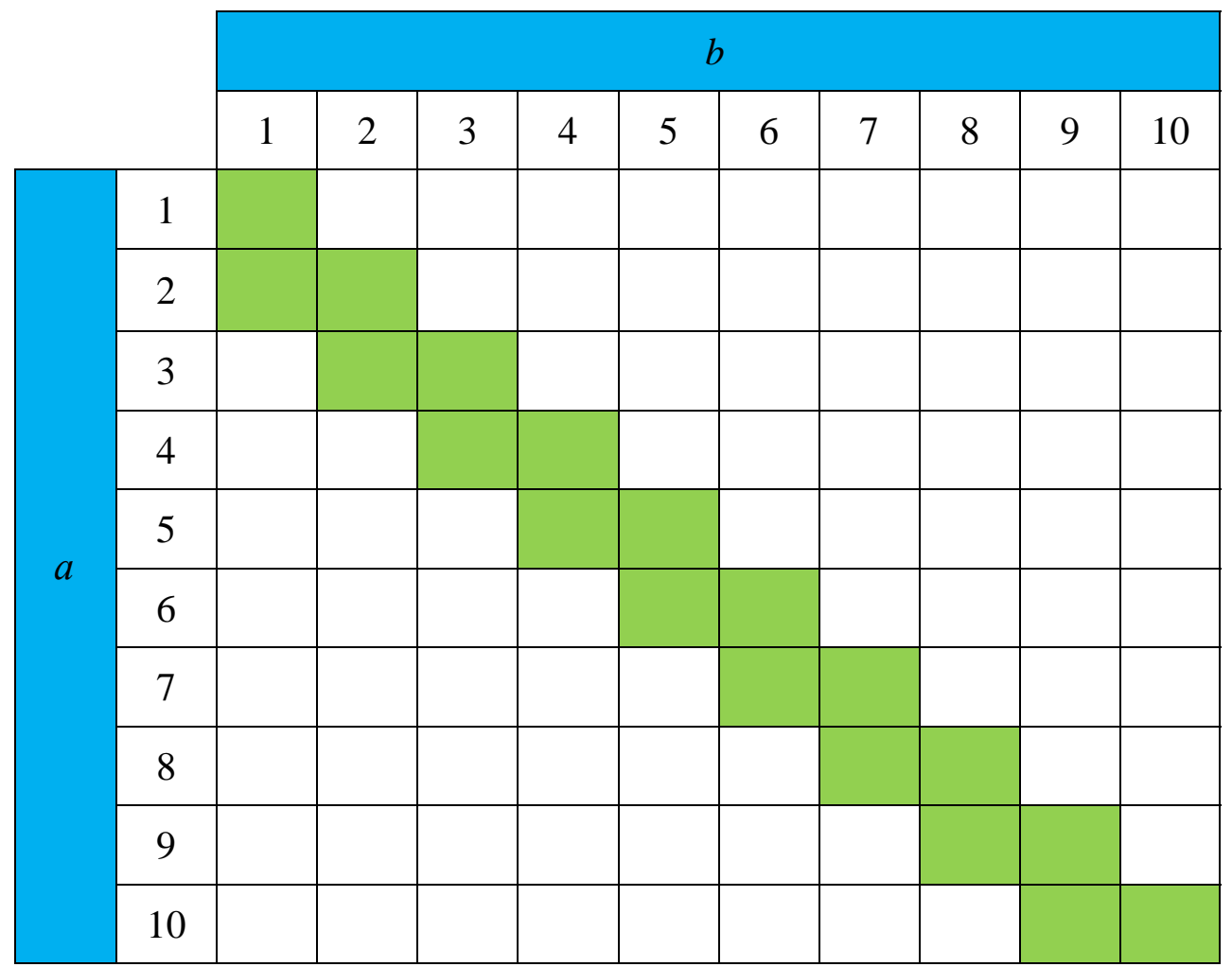

Рисунок 23 - Сводная таблица применимости функции $\tilde{r}$ и формулы

$$
\sum_{n=1}^{\infty} \tilde{r}\left(\sin \left(\frac{\pi\left(n-\frac{1}{2}\right)}{\left(\frac{a+b}{2}\right)}\right)\right) u_{n},
$$

для вычисления периодических значений знакопеременного ряда.

\section{ВЫВОДЫ}

Полученные аналитические формулы, алгоритмы и данные - позволяют приблизиться к решению вопроса о существовании аналитической формулы задания знака знакопеременного ряда с произвольным, но периодическим порядком знаков.

Кроме рассмотренных вариантов, остается открытым вопрос о динамически меняющемся периоде появления знаков.

\section{References}

1. Il'in VA, Pozdnyak EG (2005) Osnovy matematicheskogo analiza. Moscow: Fizmatlit. Ch.1 - 2005, 7-e izd., 648; Ch.2 - 2002, 4-e izd., 464.

2. Piskunov NS (1985) Differentsial'noe i integral'noe ischislenie. Izdatel'stvo: Moscow: Nauka, Glavnaya redaktsiya fiziko-matematicheskoy literatury, $1 \mathrm{t}-432,2 \mathrm{t}-560$.

3. Rudin U (1976) Osnovy matematicheskogo analiza. Moscow, Mir., 320.

4. Demidovich BP (1978) Zadachi i uprazhneniya po matematicheskomu analizu. Moscow. Nauka., 480. 
5. Devlin Keith (1994) Mathematics, the science of patterns: the search for order in life, mind, and the universe. Scientific American Library.

6. Kline Morris (1983) «Euler and Infinite Series». Mathematics Magazine 56 (5): 307-314. DOI:10.2307/2690371.

7. Fikhtengol'ts GM (2001) Kurs differentsial'nogo i integral'nogo ischisleniya. Moscow: FIZMATLIT, t.1 - 616; t.2 - 810; t.3 - 662.

8. Il'in VA, Pozdnyak EG (1973) Kurs matematicheskogo analiza. Moscow. Nauka, 472.

9. Kudryavtsev LD (2005) Kurs matematicheskogo analiza. Moscow: Fizmatlit, t.1 - 400; t.2 $-424$.

10. Zudilin VV (2011) Arifmeticheskie gipergeometricheskie ryady, UMN, tom 66, vypusk 2(398), 163-216. http://dx.doi.org/10.4213/rm9420

11. Davis Harry F (1989) Fourier Series and Orthogonal Functions. Dover.

12. Znakochereduyushchiysya natural'nyy ryad. http://nhpproxy.webcreatif.ch/cgibin/cgiproxy/nphproxy.cgi/111110A/http/ru.wikipedia.org/wiki/1_\%E2\%88\%92_2_+_3_\%E2\%88\%92_4_ $+\% \mathrm{E} 2 \% 80 \% \mathrm{~A} 6$ 